\title{
NORMAL AUTOMORPHISMS AND THEIR FIXED POINTS
}

\author{
BY \\ FRANKLIN HAIMO( ${ }^{(1)}$
}

1. Introduction. The elements of the centralizer $T$ of the group of inner automorphisms $J$ of a group $G$ (in the group $A$ of automorphisms of $G$ ) are called the normal automorphisms of $G$. The center $Z$ of $G$ is the set of all elements of $G$ which are fixed by each mapping from $J$. Likewise, let $B$ be the set of fixed points held in common by the mappings from $T . G / B$ is abelian, and the elements of $T$ which induce either the identity or the involution on $G / B$ form a subgroup $W$ of $T$. We shall investigate the ascending central series of $W$. Just as the ascending central series $\left\{Z_{i}\right\}$ is formed over $Z=Z_{1}$, so an ascending series is formed over $B$. Elements of $G$ lying in members of this $B$-series turn out to be fixed points for high powers of normal automorphisms. For automorphisms which induce the identity on $G / Z_{n}$, we show that the common fixed points lie in the centralizer of $Z_{n}$ in $G$.

The notation is both obvious and conventional. $G$ denotes a group with automorphism group $A$ and inner automorphism group $J$. The members of the ascending central series of $G$ are the $Z_{i}$, and the higher commutator subgroups are the $G^{(i)}$ [3]. If, say, the inner automorphism group of a group $H$, different from $G$, is to be denoted, we employ the symbols $J(H)$, and similarly for other groups or subgroups, such as $Z_{i}(H)$, associated with $H$. For a subgroup $H$ of $G$, the centralizer of $H$ in $G$ will be denoted by $C(H ; G)$. If $x, y \in G$, then $(x, y)=x^{-1} y^{-1} x y$. For normal subgroups $S$ and $T$ of a group $G, S \div T$ (following R. Baer) will be the commutator quotient of $S$ by $T$, the set of all $x \in G$ such that $(x, t) \in S$ for every $t \in T . S \div T$ is a normal subgroup of $G$. The identity map on a group is indicated by $\iota$, and the identity element of a group is to be $e$. For a homomorphism $f$ on $G$, the kernel will be written kern $f . \oplus$ denotes direct summation of groups. A periodic group is one in which each element is of finite order, and an abelian periodic group will be called a torsion group. If a periodic group $G$ has a uniform order on its elements, then $G$ is said to be uniform torsion (u.t.), and the least positive uniform order will be called [3] the exponent of $G$. A group is said to be torsionfree if it has no nontrivial elements of finite order. A complete group is one in which the $x^{n}$ form a set of generators of $G$ for each positive integer $n$. The group of integers is to be $I$; the group of rationals, $R$; the multiplicative group of nonzero rationals, $R^{*}$; and $I_{n}$ is to be the group of integers modulo $n$.

Presented to the Society, September 2, 1952; received by the editors September 3, 1953.

(1) This research was supported in part by The United States Airforce under contract No. AF18(600)-568 monitored by the Office of Scientific Research, Air Research and Development Command. 
Occasionally, we shall give $I_{n}$ its usual representation as the group of integral residue classes, modulo $n$, so that $j_{n}$ will be the residue class, modulo $n$, in which the integer $j$ lies.

2. Normal automorphisms. Let $G$ be a group, and let $H$ be a normal subgroup of $G$. Let $\alpha$ be an automorphism of $G$ such that $H$ is admissible under both $\alpha$ and $\alpha^{-1}$. That is, $\alpha(H) \subset H$ and $\alpha^{-1}(H) \subset H$. Then $\alpha$ induces an automorphism $\alpha^{\prime}$ on $G / H$ given by $\alpha^{\prime}(x H)=\alpha(x) H$. In particular, if $H$ is a characteristic subgroup of $G$, then every automorphism $\alpha$ of $G$ induces an automorphism $\alpha^{\prime}$ on $G / H$. If $\alpha$ induces the identity automorphism $\iota$ on $G / Z_{1}, \alpha$ is called a normal automorphism (sometimes center [3] or central [1] automorphism) of $G$, and $\alpha(x) \equiv x \bmod Z_{1}$ for every $x \in G$. It is easy to see that $\alpha \in A$ is normal if, and only if, $(x, \alpha(y))=(x, y)$ for every $x, y \in G$. Let $T_{1}$ be the set of all normal automorphisms of $G$. If automorphism composition is interpreted as a multiplication, $T_{1}$ becomes a subgroup of the automorphism group $A$ of $G$ with $\iota$ as its identity, and $T_{1}$ is normal in $A$. It is well known that $T_{1}=C(J ; A)$ [3]. An endomorphism $\gamma$ of $G$ for which $\gamma(G) \subset Z_{1}$ is called a central endomorphism. If $\alpha \in T_{1}, \alpha(x)=x \gamma(x)$ for every $x \in G$, where $\gamma$ is a central endomorphism of $G$ with the further property (A) that to each $y \in G$, there exists a unique $g=g(y ; \gamma) \in G$ with $\gamma(g)=g^{-1} y$. We might write $\alpha=\imath+\gamma$. If, conversely, $\gamma$ is a central endomorphism with (A), then the mapping $\alpha$, defined by $\alpha=\imath+\gamma$, is in $T_{1}$.

Let $G$ be a group for which $Z_{2} \neq Z_{1}$. If $u \in Z_{2}, u \in Z_{1}$, then the mapping $\gamma_{u}$ given by $\gamma_{u}(x)=(x, u)$ is readily seen to be a central endomorphism of $G$. These $\gamma_{u}$ will be called the Grün endomorphisms of $G$. If $y \in G$, then $\gamma_{u}\left(u y u^{-1}\right)$ $=\left(u y u^{-1}\right)^{-1} y$ so that $\gamma_{u}(x)=x^{-1} y$ has the solution $x=u y u^{-1}$. If, conversely, $x$ is any solution of $\gamma_{u}(x)=x^{-1} y$, then $u^{-1} x u=y$ so that $x=u y u^{-1}$; and the solution is unique, establishing (A). Hence $\alpha_{u}$, a mapping defined by $\alpha_{u}(x)=x \gamma_{u}(x)$ $=u^{-1} x u=\tau_{u}(x)$, is in $T_{1}$. Suppose, conversely, that $\alpha \in T_{1} \cap J$. Let $\alpha=\tau_{u}$. Then $u^{-1} x u \equiv x \bmod Z_{1}$ for every $x \in G$, so that $u \in Z_{2}$ and $\alpha=\alpha_{u}$. We state

Lемма 1. $T_{1} \cap J \cong Z_{1}(J)$, and the elements of the former are in one-to-one correspondence with the Grün endomorphisms of $G$.

For endomorphisms $\gamma_{\alpha}$ and $\gamma_{\beta}$ satisfying (A), note that $\gamma_{\alpha}(G) \subset \gamma_{\beta}(G)$ implies $\gamma_{\alpha \beta}(G) \subset \gamma_{\beta}(G)$, so that $\gamma_{\alpha^{n}}(G) \subset \gamma_{\alpha}(G)(n=1,2,3 \cdots) . \gamma_{\iota}$ is the trivial endomorphism $\left(\gamma_{\iota}(x)=e\right.$ for every $\left.x \in G\right)$, and $\gamma_{\iota}(G) \subset \gamma_{\alpha}(G)$ for every $\alpha \in T_{1}$.

Lemma 2. For $\alpha \in T_{1}, \alpha\left(\gamma_{\alpha}(G)\right)=\gamma_{\alpha}(G)=\alpha\left(\gamma_{\alpha}-1(G)\right)$.

Proof. For $x \in G, \alpha(\alpha(x))=\alpha(x) \alpha\left(\gamma_{\alpha}(x)\right)$, so that $\alpha\left(\gamma_{\alpha}(x)\right) \in \gamma_{\alpha}(G)$. Hence $\alpha\left(\gamma_{\alpha}(G)\right) \subset \gamma_{\alpha}(G) . \quad \alpha^{-1}(x)=x \gamma_{\alpha}-1(x) \quad$ implies that $x=\alpha(x) \alpha\left(\gamma_{\alpha}-1(x)\right)$ $=x \gamma_{\alpha}(x) \alpha\left(\gamma_{\alpha^{-1}}(x)\right)$, whence $\gamma_{\alpha}(x)=\alpha\left(\gamma_{\alpha^{-1}}\left(x^{-1}\right)\right)$; and $\gamma_{\alpha}(G) \subset \alpha\left(\gamma_{\alpha}^{-1}(G)\right)$. Replacing $x$ by $x^{-1}$, we have $\gamma_{\alpha}\left(x^{-1}\right)=\alpha\left(\gamma_{\alpha}^{-1}(x)\right)$. There exists $y \in G$ such that $\alpha(y)=\gamma_{\alpha}\left(x^{-1}\right)=y \gamma_{\alpha}(y)$. Since $\gamma_{\alpha}(G)$ is a subgroup of $G, y \in \gamma_{\alpha}(G)$. Thus, 
$\alpha\left(\gamma_{\alpha^{-1}}(x)\right)=\alpha(y)$ where $y \in \gamma_{\alpha}(G)$, so that $\alpha\left(\gamma_{\alpha^{-1}}(G)\right) \subset \alpha\left(\gamma_{\alpha}(G)\right)$.

3. The common fixed points. The subgroup $Z_{1}$, the center of $G$, is the set of all elements of $G$ which are fixed by each inner automorphism $\tau_{y}$ of $G$. For $T_{1}=C(J ; A)$, the set analogous to $Z_{1}$ is $B_{1}$, where $x \in B_{1}$ if, and only if, $\alpha(x)=x$ for every $\alpha \in T_{1}$. If $F(\alpha)$ is the set of the fixed points of $\alpha \in T_{1}$, then $F(\alpha)$ is a normal subgroup of $G$. Since $B_{1}=\bigcap F(\alpha)$, where the cross-cut is taken over all $\alpha \in T_{1}, B_{1}$ is likewise a normal subgroup of $G$. Now $F(\alpha)$ $=$ kern $\gamma_{\alpha}$, and $\gamma_{\alpha}(G)$ is abelian. Thus $F(\alpha) \supset G^{\prime}$, the derivative of $G$, for every $\alpha \in T_{1}$, and $B_{1} \supset G^{\prime}$. This shows that $G / B_{1}$ is abelian and that if $B_{1}=(e)$, then $G$ is abelian.

Lemma 3. $G^{\prime} \subset B_{1} \subset C\left(Z_{2} ; G\right)$.

Proof. If $x \in B_{1}, \gamma_{u}(x)=e$ for every Grün endomorphism $\gamma_{u}, u \in Z_{2}$. Consequently $x$ commutes with every such $u$.

Corollary. If $C\left(Z_{2} ; G\right)=Z_{1}$, then $G$ is of class 2 .

Suppose that $H$ is a characteristic subgroup of $G$, that $J(H ; G)$ is the set of all inner automorphisms $\tau_{v}$ of $G$ where $v \in H$ (where $\tau_{v}(x)=v^{-1} x v$ ), and that $Q(H ; G)=Q(H)$ is the set of all automorphisms of $G$ such that $\alpha \in Q(H)$ induces the identity automorphism on $G / H$. For instance, $J(G ; G)=J$, and $Q\left(Z_{1} ; G\right)=T_{1} . J(H ; G)$ is a normal subgroup of $Q(H ; G)$. Let $F=F(Q(H ; G))$ be the fixed points common to all mappings in $Q(H ; G)$, and let $F^{*}$ $=F(J(H ; G))$ be the fixed points common to all mappings in $J(H ; G)$. $F^{*} \supset F$. But $F^{*}=C(H ; G)$, so that $F(Q(H ; G)) \subset C(H ; G)$. This general result will be used later to establish a variation of Lemma 3.

$G=B_{1}$ if, and only if, $G$ has no proper normal automorphisms. By Lemma 3, $G=B_{1}$ implies $G=C\left(Z_{2} ; G\right)$ so that every element of $G$ commutes with every element of $Z_{2}$, and $Z_{2} \subset Z_{1}$. Hence the ascending central series of $G$ breaks off with $Z_{1}$ if $G=B_{1}$. Likewise, Lemma 3 has the following obvious

Corollary. $G$ is of class 2 if, and only if, $B_{1} \subset Z_{1}$.

In particular, $G^{\prime} \subset Z_{1}$ if, and only if, $B_{1} \subset Z_{1}$.

LemMA 4.(a) If $T_{1}$ is finite and if $Z_{1}$ is a torsion group, then $G / B_{1}$ is a torsion group. (b) If $Z_{1}$ is u.t., then $G / B_{1}$ is u.t. and $\exp G / B_{1} \mid \exp Z_{1}$. (c) If $Z_{1}$ is torsion-free, then so is $G / B_{1}$.

Proof. (a) For $x \in G$ and $\alpha \in T_{1}, \alpha(x)=x \gamma_{\alpha}(x)$, where $\gamma_{\alpha}(x) \in Z_{1}$. There exists a least positive integer $n=n(x ; \alpha)$ such that $\gamma_{\alpha}\left(x^{n}\right)=e$, since $Z_{1}$ is a torsion group. Since $T_{1}$ is finite, we can form $n(x)$, the least common multiple of all such $n(x ; \alpha)$. For $\alpha \in T_{1}, \alpha\left(x^{n(x)}\right)=x^{n(x)}$ so that $x^{n(x)} \in B_{1}$, and $G / B_{1}$ is a torsion group. (b) has a proof which is an obvious modification of the proof of (a). (c) Suppose that $Z_{1}$ is torsion-free and that $x^{n} \in B_{1}$. Then for $\alpha \in T_{1}$, $\alpha\left(x^{n}\right)=x^{n}$. But $\alpha(x)=x \gamma_{\alpha}(x)$, so that $\gamma_{\alpha}\left(x^{n}\right)=e$. Since $\gamma_{\alpha}(x)$ is not a periodic 
element, $\gamma_{\alpha}(x)=e$ and $x \in B_{1}$. Hence $G / B_{1}$ is torsion-free.

Lemma 5. If $G / B_{1}$ is complete, then each $\gamma_{\alpha}(G)$ is complete; and if, in addition, $Z_{1}$ is torsion-free, $G / B_{1}$ and each $\gamma_{\alpha}(G)$ are direct sums of copies of $R$, the additive group of the rationals.

Proof. For $z \in \gamma_{\alpha}(G)$, there exist $x \in G$ and $\alpha \in T_{1}$ with $\alpha(x)=x z$. Since $G / B_{1}$ is complete, for each positive integer $n$ there exists $y \in G$ with $x$ $\equiv y^{n} \bmod B_{1} . \alpha\left(y^{n} x^{-1}\right)=y^{n} x^{-1}=y^{n} \gamma_{\alpha}\left(y^{n}\right) x^{-1} z^{-1}=y^{n} x^{-1} \gamma_{\alpha}\left(y^{n}\right) z^{-1}$. Hence $\gamma_{\alpha}\left(y^{n}\right)$ $=z$, and $\left[\gamma_{\alpha}(y)\right]^{n}=z$. Since $\gamma_{\alpha}(G)$ is abelian, this is enough to show that it complete. If, in addition, $Z_{1}$ is torsion-free, then Lemma 4(c) shows that $G / B_{1}$ is torsion-free. Also each $\gamma_{\alpha}(G)$ is torsion-free. But torsion-free, complete abelian groups are direct sums of copies of $R$.

4. Automorphisms induced on $G / B_{1}$. Since $B_{1}$ is admissible under each normal automorphism of $G$, each such automorphism induces an automorphism on $G / B_{1}$. (See, however, [1] where $G / G^{\prime}$ for finite $G$ is discussed instead.) If $\alpha \in T_{1}$ induces the identity on $G / B_{1}$, then $\alpha(x)=x \gamma_{\alpha}(x) \equiv x \bmod B_{1}$ so that $\gamma_{\alpha}(G) \subset B_{1}$. Conversely, if $\alpha \in T_{1}$ and if $\gamma_{\alpha}(G) \subset B_{1}$, then the induced automorphism $\alpha^{\prime}$ has the property $\alpha^{\prime}\left(x B_{1}\right)=\alpha(x) B_{1}=x \gamma_{\alpha}(x) B_{1}=x B_{1}$ for every $x B_{1} \in G / B_{1}$. Thus, a necessary and sufficient condition that $\alpha \in T_{1}$ induce $\iota$ on $G / B_{1}$ is that $\gamma_{\alpha}(G) \subset B_{1}$. Let the set of all such $\alpha \in T_{1}$ be denoted by $V_{1}$. By a well known result $[3$, p. 78] on automorphisms which leave a normal subgroup $H$ and the factor group $G / H$ point-wise fixed, $V_{1}$ is an abelian group under automorphism composition. $V_{1}$ is a normal subgroup of $T_{1}$. For, if $\alpha \in V_{1}, \beta \in T_{1}$, then $\beta^{-1} \alpha \beta(x)=\beta^{-1} \alpha\left(x \gamma_{\beta}(x)\right)=\beta^{-1}\left(x \gamma_{\beta}(x) b c\right)=x b c$ where $b$, $c \in B_{1}$, and $\alpha(x)=x b, \alpha \gamma_{\beta}(x)=\gamma_{\beta}(x) c$. This makes $V_{1}$ normal in $T_{1}$. Moreover, $\alpha^{-1} \beta^{-1} \alpha \beta(x)=x c$, and we have

Lemma 6. If $\alpha \in V_{1}$ and if $\beta \in T_{1}$, then $\gamma_{(\alpha, \beta)}=\gamma_{\alpha} \gamma_{\beta}$.

Since $G / B_{1}$ is an abelian group, it has the automorphism $\omega$ given by $\omega(y)=y^{-1}$ for every $y \in G / B_{1} . \omega^{2}=\iota$, and $\omega$ is called the involution automorphism.

Lemma 7. (a) If $\alpha \in T_{1}$ induces the involution automorphism on $G / B_{1}$, then $\alpha$ induces the involution automorphism on $\gamma_{\alpha}(G)$. (b) $\alpha \in T_{1}$ induces $\omega$ on $G / B_{1}$ if, and only if, $\gamma_{\beta} \gamma_{\alpha}(x)=\gamma_{\beta}\left(x^{-2}\right)$ for every $x \in G$ and for every $\beta \in T_{1}$.

Proof. (a) For $x \in G, \alpha(x)=\dot{x} \gamma_{\alpha}(x) \equiv x^{-1} \bmod B_{1}$ so that $x^{2} \gamma_{\alpha}(x) \in B_{1}$ and $\gamma_{\alpha}\left(x^{2}\right) \gamma_{\alpha}\left(\gamma_{\alpha}(x)\right)=e$. Thus $\gamma_{\alpha}(x) \alpha\left(\gamma_{\alpha}(x)\right)=e$, so that $\alpha\left(\gamma_{\alpha}(x)\right)=\gamma_{\alpha}\left(x^{-1}\right)$, and $\alpha$ induces $\omega$ on $\gamma_{\alpha}(G)$. (b) $\alpha$ induces $\omega$ on $G / B_{1}$ if, and only if, $x^{2} \gamma_{\alpha}(x) \in B_{1}$ for every $x \in G$. For $\beta \in T_{1}, \gamma_{\beta}\left(x^{2} \gamma_{\alpha}(x)\right)=e$ so that $\gamma_{\beta} \gamma_{\alpha}(x)=\gamma_{\beta}\left(x^{-2}\right)$. Conversely, if $\gamma_{\beta} \gamma_{\alpha}(x)=\gamma_{\beta}\left(x^{-2}\right)$ for every $\beta \in T_{1}$, then $x^{2} \gamma_{\alpha}(x) \in B_{1}$.

If we let $W_{1}$ be the set of all $\alpha \in T_{1}$ which induce either $\iota$ or $\omega$ on $G / B_{1}$, then $W_{1}$ is a group under automorphism composition. Let the set of those elements of $W_{1}$ which are not in $V_{1}$ be denoted by $W_{1}^{*}$. Assume, for the pres- 
ent, that this set is nonvoid. It is easy to verify that the elements of $W_{1}^{*}$ are carried into elements of $W_{1}^{*}$ by the inner automorphisms of the group $T_{1}$, so that $W_{1}$ is a normal subgroup of $T_{1}$. The index $\left[W_{1}: V_{1}\right]=2$, and $W_{1} / V_{1} \cong I_{2}$; for, if $\alpha, \beta \in W_{1}^{*}$, then $\alpha^{-1} \beta(x)=\alpha^{-1}\left(x^{-1} b\right)=\alpha^{-1}\left(x^{-1}\right) b$, where $b \in B_{1}$. Since $\alpha(x)=x^{-1} c$ (where $\left.c \in B_{1}\right), \alpha^{-1}\left(x^{-1}\right)=x c^{-1}$, and $\alpha^{-1} \beta(x) \equiv x \bmod B_{1}$ so that $\alpha^{-1} \beta \in V_{1}$.

THEOREM 1. (a) For a group $G, W_{1}$ is $j$-nilpotent for a given positive integer $j$, or $Z_{j}\left(W_{1}\right)$ is included properly in $V_{1}$. (b) If $W_{1}^{*}$ is nonvoid, then $\alpha \in Z_{j}\left(W_{1}\right)$ $\cap V_{1}$ if, and only if, $\gamma_{\alpha}\left(x^{2^{j}}\right)=e$ for every $x \in G$ and $\alpha \in V_{1}$.

Proof. If $W_{1}^{*}$ is void, then $V_{1}=W_{1}$ and $W_{1}$ is abelian. Let us therefore assume that $W_{1}^{*}$ is nonvoid. First suppose that $j=1$, and consider $\alpha \in V_{1}$ $\cap Z_{1}\left(W_{1}\right)$. Choose $\beta \in W_{1}^{*}$. Then since $\alpha \in Z_{1}\left(W_{1}\right),(\alpha, \beta)=\imath$. Now $\gamma_{(\alpha, \beta)}(x)$ $=\gamma_{\alpha} \gamma_{\beta}(x)=\gamma_{\alpha}\left(x^{-2}\right)$, by Lemmas 6 and $7(\mathrm{~b})$. Since $\gamma_{\mathrm{l}}(x)=e$ for every $x \in G$, $\gamma_{\alpha}\left(x^{-2}\right)=e$ for every $x \in G$. Conversely, if $\gamma_{\alpha}\left(x^{2}\right)=e$ for every $x \in G$, then $\gamma_{\alpha} \gamma_{\beta}(x)=\gamma_{(\alpha, \beta)}(x)=e$ for every $x \in G$ and for every $\beta \in W_{1}^{*}$, by Lemmas 6 and 7 (b). But $\gamma_{(\alpha, \beta)}(x)=e$ for every $x \in G$ implies that $(\alpha, \beta)=\iota$ for every $\beta \in W_{1}^{*}$. Since $V_{1}$ is abelian, and since $\alpha \in V_{1}, \alpha$ is in $Z_{1}\left(W_{1}\right)$. We have verified (b) in the case $j=1$.

Suppose that there exists $\beta \in Z_{1}\left(W_{1}\right) \cap W_{1}^{*}$. Since $\left[W_{1}: V_{1}\right]=2, \beta \in Z_{1}\left(W_{1}\right)$ if, and only if, $W_{1}^{*} \subset Z_{1}\left(W_{1}\right)$. If $\alpha \in V_{1}, \beta \in Z_{1}\left(W_{1}\right) \cap W_{1}^{*}$, then $(\alpha, \beta)=\iota$ and $\gamma_{(\alpha, \beta)}(x)=\gamma_{\alpha} \gamma_{\beta}(x)=e$ for every $x \in G$, by Lemma 6 . By Lemma $7(\mathrm{~b}), \beta \in W_{1}^{*}$ implies $\gamma_{\alpha} \gamma_{\beta}(x)=\gamma_{\alpha}\left(x^{-2}\right)=e$. By (b), which has been established for the case $j=1, \alpha \in Z_{1}\left(W_{1}\right)$. Hence $W_{1}=W_{1}^{*} \cup V_{1} \subset Z_{1}\left(W_{1}\right)$. It follows that if $W_{1} \neq Z_{1}\left(W_{1}\right)$ then $Z_{1}\left(W_{1}\right) \subset V_{1}$; and this inclusion must be strict. For, if not, $Z_{1}\left(W_{1}\right)=V_{1}$ and $W_{1} / V_{1} \cong J\left(W_{1}\right)$. Since $W_{1} / V_{1} \cong I_{2}, J\left(W_{1}\right)$ is cyclic, an impossibility [2]. We have now established (a) for the case $j=1$.

Now suppose that the theorem holds for the case $j-1$. If $\beta \in W_{1}^{*}$ and if $\alpha \in V_{1} \cap Z_{j}\left(W_{1}\right),(\alpha, \beta) \in Z_{j-1}\left(W_{1}\right)$. If $x \in G,(\alpha, \beta)(x)=x \gamma_{\alpha}\left(\gamma_{\beta}(x)\right)=x \gamma_{\alpha}\left(x^{-2}\right)$. Noting that $(\alpha, \beta) \in V_{1}$ since $\gamma_{\alpha}\left(x^{-2}\right) \in B_{1}$, (b) can be applied for the case $j-1$, and $\gamma_{\alpha}\left[\left(x^{2^{j-1}}\right)^{-2}\right]=e$, whence $\gamma_{\alpha}\left(x^{2^{j}}\right)=e$ for every $x \in G$. Conversely, suppose that $\alpha \in V_{1}$ and that $\gamma_{\alpha}\left(x^{2^{j}}\right)=e$ for every $x \in G$. Choose $\beta \in W_{1}^{*} \cdot \gamma_{(\alpha, \beta)}(y)$ $=\gamma_{\alpha} \gamma_{\beta}(y)=\gamma_{\alpha}\left(y^{-2}\right)$ for every $y \in G$. Let $y=x^{2^{j-1}}$. Then $\gamma_{\alpha}\left(y^{-2}\right)=e$ by assumption, and $\gamma_{(\alpha, \beta)}\left(x^{2^{j-1}}\right)=e$ for every $x \in G$. Since $\alpha \in V_{1}$ implies $\gamma_{\alpha}\left(y^{-2}\right) \in B_{1}$, $\gamma_{(\alpha, \beta)}(y) \in B_{1}$ and $(\alpha, \beta) \in V_{1}$. By (b) for the case $j-1,(\alpha, \beta) \in Z_{j-1}\left(W_{1}\right)$ for every $\beta \in W_{1}^{*}$. If $\beta \in V_{1}$, then the fact that $V_{1}$ is abelian allows one to conclude that $(\alpha, \beta)=\iota \in Z_{j-1}\left(W_{1}\right)$. Hence $(\alpha, \beta) \in Z_{j-1}\left(W_{1}\right)$ for every $\beta \in W_{1}$, and $\alpha \in Z_{j}\left(W_{1}\right)$. This establishes (b) for the case $j$.

Since $\left[W_{1} ; V_{1}\right]=2$, the elements of $W_{1}^{*}$ all have the form $\beta \alpha$ where $\alpha \in V_{1}$. Suppose now that $\beta \in Z_{j}\left(W_{1}\right) \cap W_{1}^{*}$ and that $\alpha$ and $\delta$ are elements of $V_{1}$. $\beta \alpha \beta \delta \equiv \beta \alpha \delta \beta \equiv \beta \delta \alpha \beta \equiv \beta \delta \beta \alpha \bmod Z_{j-1}\left(W_{1}\right)$. Likewise, $\beta \alpha \delta \equiv \delta \beta \alpha \bmod Z_{j-1}\left(W_{1}\right)$. Hence if $Z_{j}\left(W_{1}\right) \cap W_{1}^{*}$ is nonvoid, then $W_{1}^{*} \subset Z_{j}\left(W_{1}\right)$. If $\alpha \in V_{1}, \beta \in Z_{j}\left(W_{1}\right)$ $\cap W_{1}{ }^{*}$, then $(\alpha, \beta) \in Z_{j-1}\left(W_{1}\right)$ and $\gamma_{(\alpha, \beta)}(x)=\gamma_{\alpha} \gamma_{\beta}(x)=\gamma_{\alpha}\left(x^{-2}\right)$. As above, 
$\alpha \in V_{1}$ implies $(\alpha, \beta) \in V_{1}$ so that (b) for the case $j-1$ applies, and $\gamma_{\alpha}\left[x^{\left(2^{j-1}\right)}\right]^{-2}$ $=e$. Thus (b) for the case $j$, established above, places $\alpha \in Z_{j}\left(W_{1}\right) . W_{1}=W_{1}^{*}$ $\cup V_{1} \subset Z_{j}\left(W_{1}\right)$, and $W_{1}=Z_{j}\left(W_{1}\right)$.

If $W_{1} \neq Z_{j}\left(W_{1}\right)$, the above shows that $Z_{j}\left(W_{1}\right) \subset V_{1}$. If the inclusion is not strict, then $Z_{j}\left(W_{1}\right)=V_{1}$ and $I_{2} \cong W_{1} / V_{1} \cong J\left(W_{1} / Z_{j-1}\left(W_{1}\right)\right)$, an impossibility [2]. This completes the proof of the theorem.

CoRollary 1. Let $G$ be a group for which $W_{1}^{*}$ is nonvoid. (a) If $Z_{1}$ is u.t. with exponent dividing $2^{j}$ (where $j>1$ ), then $W_{1}$ is nilpotent of class $\leqq j$. (b) If $Z_{1}$ is torsion-free and if $V_{1}$ is nontrivial, then $W_{1}$ is non-nilpotent.

Proof. (a) For $\alpha, \zeta \in V_{1},(\alpha, \zeta)=\iota$ since $V_{1}$ is abelian. Choose $\beta \in W_{1}^{*}$. Since $\left[W_{1}: V_{1}\right]=2, W_{1}^{*}$ is the coset of $V_{1}$ in $W_{1}$ which contains $\beta \alpha$. For $x \in G$, $\alpha(x)=x b, \zeta(x)=x d$, and $\beta(x)=x^{-1} c$, where $b, d \in Z_{1} \cap B_{1}$ and $c \in B_{1} .(\beta \alpha, \zeta)(x)$ $=\alpha^{-1} \beta^{-1} \zeta^{-1} \beta \alpha \zeta(x)=\alpha^{-1} \beta^{-1} \zeta^{-1} \beta(x b d)=\alpha^{-1} \beta^{-1} \zeta^{-1}\left(x^{-1} c b d\right)=\alpha^{-1} \beta^{-1}\left(d x^{-1} c b d\right)$ $=\alpha^{-1}\left(d x c^{-1} c b d\right)=d x b^{-1} b d=x d^{2} \quad$ so that $(\beta \alpha, \zeta)=\delta_{1} \in V_{1} . \quad(\beta \alpha, \quad \beta \zeta)(x)$ $=\alpha^{-1} \beta^{-1} \zeta^{-1} \alpha \beta \zeta(x)=\alpha^{-1} \beta^{-1} \zeta^{-1} \alpha \beta(x d)=\alpha^{-1} \beta^{-1} \zeta^{-1} \alpha\left(x^{-1} c d\right)=\alpha^{-1} \beta^{-1} \zeta^{-1}\left(b^{-1} x^{-1} c d\right)$ $=\alpha^{-1} \beta^{-1}\left(b^{-1} d x^{-1} c d\right)=\alpha^{-1}\left(b^{-1} d x c^{-1} c d\right)=b^{-1} d x b^{-1} d=x\left(b^{-1} d\right)^{2}$, so that $(\beta \alpha, \beta \zeta)$ $=\delta_{2} \in V_{1}$. If $\exp Z_{1} \mid 2^{j}$, then $\delta_{i}\left(x^{2^{i-1}}\right)=x^{2^{j-1}}\left(b^{1-i} d\right)^{2^{j}}=x^{2^{j-1}}$, and $\gamma_{\delta_{i}}\left(x^{2^{j-1}}\right)=e$ for every $x \in G$. By (b) of the theorem, $\delta_{i} \in Z_{j-1}\left(W_{1}\right)$, so that $W_{1}^{\prime} \subset Z_{j-1}\left(W_{1}\right)$, and $W_{1}$ is nilpotent of class $\leqq j$. This establishes (a) of the corollary.

(b) Now suppose that $Z_{1}$ is torsion-free. By hypothesis, we can find $\alpha$ and $\zeta \in V_{1}$ and $y \in G$ with $\alpha(y) \neq \zeta(y)$, where $\alpha(y)=y b$ and $\zeta(y)=y d$. Construct $\delta_{i}$ as in part (a) of the corollary. $\delta_{i}\left(y^{2^{j-1}}\right)=y^{2^{j-1}}\left(b^{1-i} d\right)^{2^{j}}$. Since $b \neq d$, we can always adjust our notation so that $d \neq e$. Since $Z_{1}$ is torsion-free, $b \neq d$ and $d \neq e,\left(b^{1-i} d\right)^{2^{i}} \neq e$ for each positive integer $j$, so that, by (b) of the theorem, $\delta_{i} \in Z_{j-1}\left(W_{1}\right)$, and $W_{1}^{\prime} \nsubseteq Z_{j-1}\left(W_{1}\right)$ for all such $j$, and $W_{1}$ is not nilpotent.

Corollary 2. Let $Z_{1}$ be torsion-free, $V_{1}$ be nontrivial, $W_{1}^{*}$ be nonvoid and let $G$ be complete. Then $W_{1}$ has a trivial center.

Proof. By Corollary 1(b), $W_{1}$ is not nilpotent. By (a) of the theorem, $Z_{1}\left(W_{1}\right)$ is a proper subgroup of $V_{1}$. By (b) of the theorem, $\alpha \in Z_{1}\left(W_{1}\right)$ implies $x^{2} \in F(\alpha)$, the set of all fixed points of $\alpha$, for every $x \in G$. Since $G$ is complete, $G=F(\alpha)$, and $\alpha=\iota$.

It is fairly obvious that $\alpha$ and $\beta \in T_{1}$ induce the same automorphism on $G / B_{1}$ if, and only if, $\alpha \equiv \beta \bmod V_{1}$; and an equivalent condition is that $\gamma_{\alpha}(x)$ $\equiv \gamma_{\beta}(x) \bmod B_{1}$ for every $x \in G$. It follows that if $\alpha \equiv \beta \bmod V_{1}$, then there exists an endomorphism $\lambda_{\alpha, \beta}$ on $G$ into $B_{1} \cap Z_{1}$ such that (1) the kernel of $\lambda_{\alpha, \beta}$ is just $F\left(\alpha^{-1} \beta\right)=F\left(\beta^{-1} \alpha\right)$; (2) $\gamma_{\alpha}(x)=\gamma_{\beta}(x) \lambda_{\alpha . \beta}(x)$; and (3) for $g \in G, \lambda_{\alpha, \beta}(x)$ $=\beta\left(x^{-1}\right) g$ has a unique solution $x=x(g) \in G$. Conversely, if $\lambda$ is an endomorphism of $G$ into $B_{1} \cap Z_{1}$, if $\beta \in T_{1}$ and if $\lambda(x)=\beta\left(x^{-1}\right) g$ has a unique solution $x=x(g)$ for every $g \in G$, then the mapping $\alpha$ defined by $\alpha(x)=\beta(x) \lambda(x)$ is a normal automorphism of $G$ such that $\alpha \equiv \beta \bmod V_{1}$ and such that $\lambda=\lambda_{\alpha, \beta}$. We restate as follows: 
Lemma 8. If $\beta \in T_{1}$ and if $\lambda$ is an endomorphism of $G$ into $B_{1} \cap Z_{1}$, then $\beta+\lambda \in T_{1}$ with $\beta+\lambda \equiv \beta \bmod V_{1}$ if, and only if, $\iota+\beta^{-1} \lambda \in T_{1}$.

Recall that $\tau_{x}(y)=x^{-1} y x$.

Lemma 9. (a) $\alpha \in W_{1}^{*}$ implies that $\tau_{x} \alpha^{-1}(x)=\alpha(x)$ for every $x \in G^{\text {. }}$ (b) $\alpha \in T_{1}$ and $\alpha^{2}=\iota$ imply that $\alpha$ induces $\omega$ on $\gamma_{\alpha}(G)$. If, in addition, $\alpha \in Z_{1}\left(W_{1}\right)$ $\cap V_{1}$ and if $W_{1}^{*}$ is nonvoid, then $\gamma_{\alpha}(G) \subset$ kern $\gamma_{\alpha}$.

Proof. (a) is immediate. As for (b), $\alpha \in T_{1}$ implies that $\alpha(x)=x \gamma_{\alpha}(x)$ and $\alpha^{-1}(x)=x \alpha^{-1}\left(\gamma_{\alpha}\left(x^{-1}\right)\right)=x \gamma_{\alpha^{-1}}(x)$. Hence $\alpha^{-1}\left(\gamma_{\alpha}\left(x^{-1}\right)\right)=\gamma_{\alpha^{-1}}(x)$, or $\alpha\left(\gamma_{\alpha}(x)\right)$ $=\gamma_{\alpha}\left(x^{-1}\right)$, since $\alpha^{-1}=\alpha$, and $\alpha$ induces $\omega$ on $\gamma_{\alpha}(G)$. From this, $\gamma_{\alpha}(x) \gamma_{\alpha}^{2}(x)$ $=\gamma_{\alpha}\left(x^{-1}\right)$, and $\gamma_{\alpha}\left(x^{-2}\right)=\gamma_{\alpha}^{2}(x)$. By Theorem $1(\mathrm{~b}), \gamma_{\alpha}\left(x^{-2}\right)=e$, so that $\gamma_{\alpha}^{2}(x)=e$ and $\gamma_{\alpha}(G) \subset$ kern $\gamma_{\alpha}$.

5. The $B$-series. $G / B_{1}(G)$ is an abelian group so that all of its automorphisms are normal. Define $B_{2}(G)$ as the complete inverse image in $G$ of $B_{1}\left(G / B_{1}(G)\right)$ under the natural homomorphism of $G$ onto $G / B_{1}(G)$. In general, suppose that $B_{j}(G)$ is defined. Then $B_{j+1} / B_{j} \cong B_{1}\left(G / B_{j}\right)$. We let $B_{0}(G)=(e)$. Each $B_{j}$ is a normal subgroup of $G$, and $i \leqq j$ implies that $B_{i} \subset B_{j}$, so that the $B$-series ascends monotonically in its index. Each $G / B_{j}$ is abelian $(j>0)$, and $B_{j+1} / B_{j}$ is the set of elements of $G / B_{j}$ which are each fixed by all automorphisms of $G / B_{j}(j>0)$. If $B_{j+1}=B_{j}$, then for all $k \geqq j, B_{k}=B_{j}$.

Leмma 10. The $B$-series breaks off at $B_{1}$ if any one of the following holds:

(a) $G / B_{1}$ has no elements of order 2 .

(b) $Z_{1}$ is torsion-free, or $Z_{1}$ has no elements of order 2 or no $\gamma_{\alpha}(G)$, for $\alpha \in T_{1}$, has elements of order 2 .

(c) To each $x B_{1}$ in $G / B_{1}$, there exists an automorphism $\theta=\theta_{x}$, such that $\theta\left(x B_{1}\right) \neq x B_{1}$.

(d) To each $x \in G$, there exists $\alpha=\alpha_{x} \in A$ such that $\alpha$ induces an automorphism on $G / B_{1}$, and $\alpha(x) \neq x \bmod B_{1}$.

(e) The equation $\xi^{2}=\alpha$, for $\alpha \in T_{1}$, always has a solution in $T_{1}$.

Proof. (a) Since $G / B_{1}$ is abelian, it has the involution automorphism $\omega$. If $g B_{1} \in B_{2} / B_{1}$, then $\omega\left(g B_{1}\right)=g B_{1}=g^{-1} B_{1}$, and $g^{2} \in B_{1}$. Since $G / B_{1}$ has no elements of order 2, $g \in B_{1}$ and $B_{2} \subset B_{1}$. (b) For $x^{2} \in B_{1}$ and $\alpha \in T_{1}, \alpha\left(x^{2}\right)=x^{2}$ $=x^{2} \gamma_{\alpha}\left(x^{2}\right)$, and $\gamma_{\alpha}\left(x^{2}\right)=e$. Since $\gamma_{\alpha}(G)$ has no elements of order $2, \gamma_{\alpha}(x)=e$ and $x \in B_{1}$. Hence $G / B_{1}$ has no elements of order 2 , and (a) applies. (c) There is no fixed point common to all automorphisms of $G / B_{1}$, so that $B_{2} / B_{1}$ is trivial, and $B_{2}=B_{1}$. (d) $\alpha$ induces $\alpha^{\prime}$, an automorphism on $G / B_{1} \cdot \alpha^{\prime}\left(x B_{1}\right) \neq x B_{1}$ so that (c) can now be applied. (e) If $g \in B_{2}$, then, as we saw in the proof of (a), $g^{2} \in B_{1}$. For $\beta \in T_{1}$ there exists an induced automorphism $\beta^{\prime}$ on $G / B_{1}$. Since $g \in B_{2}, \quad \beta^{\prime}\left(g B_{1}\right)=g B_{1}=\beta(g) B_{1}$. Hence $\beta(g) \equiv g \bmod \left(Z_{1} \cap B_{1}\right)$. $\beta^{2}(g)$ $=\beta\left(g \gamma_{\beta}(g)\right)=\beta(g) \gamma_{\beta}(g)=g \gamma_{\beta}\left(g^{2}\right)$, since $\gamma_{\beta}(g) \in B_{1} \cap Z_{1}$. But $g^{2} \in B_{1}$ implies that $\gamma_{\beta}\left(g^{2}\right)=e$, so that $\beta^{2}(g)=g$. Since every $\alpha \in T_{1}$ is, by hypothesis, a square, $g \in B_{1}$, and $B_{2} \subset B_{1}$. 
6. The case $G=B_{2}$. It is obvious that $G=B_{2}$ if, and only if, $B_{1}\left(G / B_{1}\right)$ $=G / B_{1}$; that is, if, and only if, the identity is the only normal automorphism of $G / B_{1}$. Since $G / B_{1}$ is abelian, we see that $G=B_{2}$ if, and only if, $G / B_{1}$ has no proper automorphism. But this is equivalent $[2 ;$ p. 101] to

LEMmA 11. $G=B_{2}$ if, and only if $G / B_{1} \cong I_{2}$.

Since $G / B_{1} \cong I_{2}$ in this case, choose $u \in B_{2}, u \notin B_{1}$. Then to each $x \in G$, $x \notin B_{1}$, there exists $b_{x} \in B_{1}$ with $x=u b_{x}$. For $\alpha \in T_{1}, \alpha(x)=u \gamma_{\alpha}(u) b_{x}=x \gamma_{\alpha}(u)$. Hence $\alpha(x)=x \gamma_{\alpha}(u)$ if $x \notin B_{1},=x$ if $x \in B_{1}$. We note that $\gamma_{\alpha}(u) \in B_{1} \cap Z_{1}$, by the proof of Lemma 10(e). Since $u^{2} \in B_{1}$ (by the proof of Lemma 10(a)), $\gamma_{\alpha}\left(u^{2}\right)=e$. It is clear that if $\alpha, \beta \in T_{1}$ then $\gamma_{\alpha \beta}(u)=\gamma_{\alpha}(u) \gamma_{\beta}(u) . \gamma_{\alpha}(u)=e$ if, and only if, $\alpha=\imath$. Moreover, suppose that $c \in Z_{1} \cap B_{1}$ and that $c^{2}=e$. Define $\alpha$ by $\alpha(x)=x c$ if $x \in B_{1},=x$ if $x \in B_{1} . \alpha(x)=e$ if, and only if, $x=e$. If $y \in B_{1}$, $\alpha(y)=y$; and if $y \in G, y \notin B_{1}$, then $\alpha\left(y c^{-1}\right)=y c^{-1} c=y$. For $x, y \in B_{1}, \alpha(x y)$ $=\alpha(x) \alpha(y)$. If $x \in B_{1}, x=u b_{x} . \quad \alpha(x)=u b_{x} c=x c$. For $y \in B_{1}, \alpha(x y)=x y c$ $=\alpha(x) \alpha(y)$. If $y \notin B_{1}$, then $y=u b_{y}$ and $\alpha(y)=y c . \alpha(x y)=\alpha\left(u^{2} b_{x} b_{y}\right)$. But $u^{2} \in B_{1}$, and $b_{x}, b_{y} \in B_{1}$. By the case already established for factors in $B_{1}, \alpha(x y)$ $=u^{2} b_{x} b_{y}=x y=x c y c=\alpha(x) \alpha(y)$, since $c^{2}=e$. It is thus seen that $\alpha$ is an automorphism of $G$ and that $\alpha \in T_{1} \cap V_{1}$ (since $\alpha$ induces the identity on $G / Z_{1}$ and on $G / B_{1}$ ). Let $K_{1}$ be the subgroup of $B_{1} \cap Z_{1}$ generated by the elements of order 2 of that group. We have proved

Theorem 2. If $G=B_{2}$, then $T_{1}$ is an elementary abelian group with exponent 2 , and $T_{1}=V_{1} \cong K_{1}$.

Corollary. If $G=B_{2}$ and if $\alpha \in T_{1}, \alpha \neq \iota$, then $\gamma_{\alpha}(G) \cong I_{2}$.

Proof. By the proof of the theorem, kern $\gamma_{\alpha}=B_{1}$. Apply Lemma 11 .

7. Some properties of the $B$-series.

LEMma 12. $B_{n+1}(G) / B_{1}(G) \cong B_{n}\left(G / B_{1}(G)\right)$.

Proof. The lemma is valid for $n=1$. Suppose that it is true for the case $j-1$. Then $B_{1}\left(\left(G / B_{1}\right) /\left(B_{j} / B_{1}\right)\right) \cong\left(B_{j}\left(G / B_{1}\right)\right) /\left(B_{j} / B_{1}\right)$ since $B_{j} / B_{1}$ $\cong B_{j-1}\left(G / B_{1}\right)$, by the induction hypothesis. But $B_{1}\left(\left(G / B_{1}\right) /\left(B_{j} / B_{1}\right)\right)$ $\cong B_{1}\left(G / B_{j}\right) \cong B_{j+1} / B_{j} \cong\left(B_{j+1} / B_{1}\right) /\left(B_{j} / B_{1}\right)$. Hence $B_{j}\left(G / B_{1}\right) \cong B_{j+1} / B_{1}$.

We say that $G$ is $B$-nilpotent of $B$-class $n$ (or $n$-B-nilpotent) if $G=B_{n}$.

Corollary. Suppose that $G$ is not $n$-B-nilpotent. The following are equivalent: (a) $G$ is (n+1)-B-nilpotent. (b) $G / B_{1}$ is $n$-B-nilpotent. (c) $G / B_{n} \cong I_{2}$.

Proof. The equivalence of (a) and (b) follows from the lemma. $G / B_{n} \cong I_{2}$ if, and only if, $B_{1}\left(G / B_{n}\right)=G / B_{n}$. But $B_{1}\left(G / B_{n}\right) \cong B_{n+1} / B_{n} \cong G / B_{n}$ if, and only if, $G=B_{n+1}$.

Lemma 13. Let $G$ be a group which is $(n+1)-B$-nilpotent but not $n-B$ nilpotent, and suppose that $Z_{k} \supset B_{n}$ and that $Z_{k-1} \supset B_{n}$. Then $G$ is k-nilpotent. 
Proof. $G=B_{n+1}$ implies that $G / B_{n} \cong I_{2}$, by Lemma 12, Corollary. Since $G / Z_{k} \cong\left(G / B_{n}\right) /\left(Z_{k} / B_{n}\right), G / Z_{k}$ must be isomorphic to $I_{2}$ or $(e)$, the only possible homomorphic images of $G / B_{n} \cong I_{2}$. But $G / Z_{k} \cong J\left(G / Z_{k-1}\right)$. Since the group of inner automorphisms of a group cannot be a nontrivial cyclic group, $G / Z_{k} \cong(e)$, and $G=Z_{k}$.

Lemma 14. If $G$ is $n$-B-nilpotent where $n \geqq 2$, then $\alpha\left(\gamma_{\alpha}(x)\right) \equiv \gamma_{\alpha}\left(x^{-1}\right)$ $\bmod B_{n-2}$, for every $\alpha \in T_{1}$ and for every $x \in G$.

Proof. First consider the case $n=2$. If $G=B_{2}$, we see, from the discussion before Theorem 2, that $\alpha \in T_{1}$ implies that $\gamma_{\alpha}(x)=\gamma_{\alpha}(u)$ if $x \notin B_{1},=e$ if $x \in B_{1}$. Here, $u$ is a representative of the non-unity coset of $B_{1}$ in $G$. Since $\gamma_{\alpha}\left(u^{2}\right)=e$ and $\gamma_{\alpha}(u) \in B_{1}$, we obtain $\gamma_{\alpha}(x) \alpha\left(\gamma_{\alpha}(x)\right)=\gamma_{\alpha}(u) \alpha\left(\gamma_{\alpha}(u)\right)=\left[\gamma_{\alpha}(u)\right]^{2}$ $=e$ if $x \notin B_{1}$. If $x \in B_{1}$, the calculation still gives $e$. Recalling that $B_{0}=(e)$, we see that the lemma is established for $n=2$.

Suppose that the lemma holds for the case $n-1$. Since $G=B_{n}, G / B_{1}$ is $(n-1)-B$-nilpotent, by Lemma 12. For $\alpha \in T_{1}$, consider the induced automorphism $\alpha^{\prime}$ on the abelian group $G / B_{1}$. By the induction assumption, if $\alpha^{\prime}\left(x B_{1}\right)=\left(x B_{1}\right)\left(z B_{1}\right)$, then $z B_{1} \alpha^{\prime}\left(z B_{1}\right) \in B_{n-3}\left(G / B_{1}\right)$. Now $\alpha(x)=x \gamma_{\alpha}(x)$, so that $\alpha^{\prime}\left(x B_{1}\right)=x \gamma_{\alpha}(x) B_{1}$, and $x z \equiv x \gamma_{\alpha}(x) \bmod B_{1}$. Hence $z \equiv \gamma_{\alpha}(x) \bmod B_{1}$ so that $z B_{1}=\gamma_{\alpha}(x) B_{1}$. A substitution shows that $\gamma_{\alpha}(x) B_{1} \alpha^{\prime}\left(\gamma_{\alpha}(x) B_{1}\right)=\gamma_{\alpha}(x)$ $\cdot \alpha\left(\gamma_{\alpha}(x)\right) B_{1} \in B_{n-3}\left(G / B_{1}\right)$. But $B_{n-3}\left(G / B_{1}\right) \cong B_{n-2} / B_{1}$, by Lemma 12 . From this we can conclude that $\gamma_{\alpha}(x) \alpha\left(\gamma_{\alpha}(x)\right) \in B_{n-2}$ for every $\alpha \in T_{1}$ and for every $x \in G$. The lemma is established.

Corollary 1. If $G=B_{n}, n \geqq 2$, and if $\alpha \in T_{1}$, then $\alpha^{2}(x) \equiv x \bmod \left(Z_{1} \cap B_{n-2}\right)$ for every $x \in G$.

Proof. $\alpha(x)=x \gamma_{\alpha}(x)$ implies that $\alpha^{2}(x)=x \gamma_{\alpha}(x) \alpha\left(\gamma_{\alpha}(x)\right)$. By the lemma, $\gamma_{\alpha}(x) \alpha\left(\gamma_{\alpha}(x)\right) \in B_{n-2}$.

Corollary 2. If $G=B_{n}, n \geqq 2$, and if $\alpha \in T_{1}$ induces $\omega$ on $\gamma_{\alpha}(G)$ or on $G / B_{1}$, then $\alpha^{2}=\iota$.

Proof. If $\alpha$ induces $\omega$ on $\gamma_{\alpha}(G)$, then $\alpha\left(\gamma_{\alpha}(x)\right)=\gamma_{\alpha}\left(x^{-1}\right)$, so that, by the proof of Corollary $1, \alpha^{2}(x)=x$ for every $x \in G$. By Lemma 7(a), if $\alpha$ induces $\omega$ on $G / B_{1}$, then $\alpha$ induces $\omega$ on $\gamma_{\alpha}(G)$.

COROLlaRy 3. Let $M(\alpha)$ be the largest subgroup of $\gamma_{\alpha}(G)$ on which $\alpha$ induces the involution automorphism. If $G=B_{n}, n \geqq 2$, then $\gamma_{\alpha}$ kern $\gamma_{\alpha}{ }^{2}=M(\alpha)$, and $\gamma_{\alpha^{2}}(G)$ is an $\alpha$-admissible subgroup of $\gamma_{\alpha}(G) \cap B_{n-2}$.

Proof. $x \in \operatorname{kern} \gamma_{\alpha^{2}}$ if, and only if, $\gamma_{\alpha^{2}}(x)=\gamma_{\alpha}(x) \alpha\left(\gamma_{\alpha}(x)\right)=e$; that is, equivalently, $\alpha \gamma_{\alpha}(x)=\gamma_{\alpha}\left(x^{-1}\right)$. But the latter is equivalent to $\gamma_{\alpha}(x) \in M(\alpha)$. By Lemma 2, $\gamma_{\alpha}(G)$ is $\alpha$-admissible, so that, for given $x \in G, \alpha\left(\gamma_{\alpha}(x)\right)=\gamma_{\alpha}(y)$ for a suitable $y=y(x ; \alpha)$. Then $\gamma_{\alpha^{2}}(x)=\gamma_{\alpha}(x) \gamma_{\alpha}(y)$, and $\alpha\left(\gamma_{\alpha^{2}}(x)\right)=\alpha\left(\gamma_{\alpha}(x)\right)$ $\cdot \alpha\left(\gamma_{\alpha}(y)\right)=\gamma_{\alpha}(y) \alpha \gamma_{\alpha}(y)=\gamma_{\alpha^{2}}(y)$. This shows that $\gamma_{\alpha^{2}}(G)$ is $\alpha$-admissible. 
Lemma 15. If $x \in B_{n}, n \geqq 2$, then $x^{2^{n-1}} \in B_{1}$.

Proof. The case $n=2$ was treated in the proof of Lemma 10(a). Suppose that the lemma is valid for $n=j$. If $x \in B_{j+1}, x B_{1} \in B_{j+1} / B_{1}=B_{j}\left(G / B_{1}\right)$. By the induction assumption, $x^{2^{j-1}} B_{1} \in B_{1}\left(G / B_{1}\right)=B_{2} / B_{1}$, and $x^{2^{j-1}} \in B_{2}$. The case $n=2$ now shows that $\left[x^{2^{i-1}}\right]^{2}=x^{2^{i}} \in B_{1}$.

Corollary. $B_{n} / B_{1}$ is u.t. abelian with $\exp \left(B_{n} / B_{1}\right) \mid 2^{n-1}$, so that, for an $n$-B-nilpotent group $G, G / B_{1}$ is u.t. abelian, and an $n$-B-nilpotent group with periodic $B_{1}$ is itself periodic.

Theorem 3. If $G$ is $n$-B-nilpotent, $n \geqq 2$, then $W_{1}$ is $(n-1)$-nilpotent.

Proof. If $W_{1}^{*}$ is void, then $W_{1}=V_{1}$, an abelian group. Suppose that $W_{1}^{*}$ is nonvoid. $x \in B_{n}$ implies $x^{2^{n-1}} \in B_{1}$, by Lemma 15. If $\alpha \in V_{1}$, then $\gamma_{\alpha}\left(x^{2^{n-1}}\right)=e$ for every $x \in G$, since $G=B_{n}$. By Theorem 1 (b), $V_{1} \subset Z_{n-1}\left(W_{1}\right)$. By Theorem $1(\mathrm{a}), W_{1}$ is $(n-1)$-nilpotent.

Corollary. If $G$ with torsion-free $Z_{1}$ is $n$-B-nilpotent, then $W_{1}=V_{1}$, or $V_{1}$ is trivial, and $W_{1}$ is an elementary abelian group with exponent 2.

Proof. If $W_{1}^{*}$ is nonvoid, then Theorem 1 , Corollary $1(\mathrm{~b})$, and the present theorem show that $V_{1}$ is trivial. Since $\alpha \in W_{1}^{*}$ implies that $\alpha^{2} \in V_{1}, W_{1}$ is elementary abelian with exponent 2 .

LEMmA 16. Each $B_{n}$ is $T_{1}$-admissible, and, if $n \geqq 1, \gamma_{\alpha}\left(B_{n}\right) \subset B_{n-1}$ for every $\alpha \in T_{1}$.

Proof. $B_{1}$ is $T_{1}$-admissible. Suppose that $B_{n-1}(G)$ is $T_{1}$-admissible for every group $G$. $g \in B_{n}$ implies that $g B_{n-1} \in B_{1}\left(G / B_{n-1}\right)$. For $\alpha \in T_{1}, B_{n-1}$ is both $\alpha$ - and $\alpha^{-1}$-admissible (by the induction assumption), and $\alpha$ induces an automorphism $\alpha^{\prime}$ on the abelian group $G / B_{n-1}$. Since $g B_{n-1} \in B_{1}\left(G / B_{n-1}\right)$, $\alpha^{\prime}\left(g B_{n-1}\right)=g B_{n-1}=\alpha(g) B_{n-1}$, and $\alpha(g) \equiv g \bmod B_{n-1}$. Hence $\gamma_{\alpha}\left(B_{n}\right) \subset B_{n-1}$. Since $B_{n-1} \subset B_{n}$ and $g \in B_{n}, \alpha(g) \in B_{n}$ so that $B_{n}$ is $\alpha$-admissible.

8. Orbital elements. An element $x \in G$ is said to be $n$-orbital if $\alpha^{n}(x)=x$ for every $\alpha \in T_{1}$. Collecting these $n$-orbital elements together in a set $L_{n}$ $=L_{n}(G)$, we see that $L_{n}$ is a subgroup of $G$. Since $T_{1}=C(J ; A), L_{n}$ is normal in $G$. More generally, $L_{n}$ is $C\left(T_{1} ; A\right)$-admissible. (We shall discuss $C\left(T_{1} ; A\right)$ below.) $L_{1}=B_{1}$, and $m \mid n$ implies $L_{m} \subset L_{n}$. Thus $G^{\prime} \subset B_{1}=L_{1} \subset L_{n}$ for every positive integer $n$, and $G / L_{n}$ is abelian. From the proof of Lemma 10(e), we see that $x \in B_{2}$ implies $\alpha^{2}(x)=x$ for every $\alpha \in T_{1}$, so that $B_{2} \subset L_{2}$.

For positive integers $s \geqq t$, let $C(s, t)=s ! / t !(s-t)$ !. Consider $C=C\left(2^{n-1}, r\right)$, where $n \geqq 2$ and $r \leqq n-1$. If $r$ is odd,

$$
C=2^{n-1} s \prod_{k=1}^{(r-1) / 2}\left(\frac{2^{n-1}-2 k}{2 k}\right)
$$

where $s \in R$ is a quotient of odd integers. Let $k=2^{c k} d_{k}$, where $c_{k}$ is a non- 
negative integer, and $d_{k}$ is an odd integer. Since $r$ is odd and $\leqq 2^{n-1}, r \leqq 2^{n-1}-1$ and $k \leqq(r-1) / 2 \leqq 2^{n-2}-1$. Thus we have $c_{k} \leqq n-3$, and $\left(2^{n-1}-2 k\right) / 2 k$ $=\left(2^{n-2-c k}-d_{k}\right) / d_{k}$, a quotient of odd integers. We have proved that $r$ odd implies that $2^{n-1} \mid C\left(2^{n-1}, r\right) . C\left(2^{n-1}, r+1\right)=\left[\left(2^{n-1}-r\right) /(r+1)\right] C\left(2^{n-1}, r\right)$. For odd $r \geqq 5$, the exponent of the highest power of 2 dividing into $r+1$ is $\leqq r-2$, so that $2^{n-r+1} \mid C\left(2^{n-1}, r+1\right)$, and $2^{n-(r+1)} \mid C\left(2^{n-1}, r+1\right)$. If $r=1$, then $r+1$ $=2$, and $2^{n-2}=2^{n-(r+1)} \mid C\left(2^{n-1}, r+1\right)$. If $r=3$, then $r+1=4$, and $2^{n-3}=2^{n-r} \mid C\left(2^{n-1}, r+1\right)$. We summarize in

Lemma 17 . For $n \geqq \max (2, r+1), 2^{n-r} \mid C\left(2^{n-1}, r\right)$.

Theorem 4. $B_{n} \subset L_{m}$, where $m=2^{n-1}$.

Proof. Since the earlier cases have been treated, we assume that $n \geqq 3$. Suppose that $g \in B_{n}$ and that $\alpha \in T_{1} . \alpha^{m}(g)=\alpha^{m-1}\left(g b_{n-1}\right)$ where $b_{n-1} \in B_{n-1}$, by Lemma 16. Assume, inductively, that $\alpha^{m}(g)=\alpha^{m-k}\left[g \prod_{r=1}^{k} b_{n-r}^{C(k, r)}\right]$ where $b_{n-r} \in B_{n-r}$, and $\gamma_{\alpha}(g)=b_{n-1}, \gamma_{\alpha}\left(b_{t}\right)=b_{t-1}(t=n-k+1, n-k+2, \cdots, n-1)$. When $r>n-1$, we take $b_{n \rightarrow r}=e$. Then

$$
\alpha^{m}(g)=\alpha^{m-(k+1)}\left[g b_{n-1}^{C(k, 1)+1}\left(\prod_{r=2}^{k} b_{n-r}^{C(k, r)+C(k, r-1)}\right) b_{n-(k+1)}^{C(k, k)}\right] .
$$

But $C(k, 1)+1=C(k+1,1), C(k, r)+C(k, r-1)=C(k+1, r)$, and $C(k, k)$ $=1=C(k+1, k+1)$. Thus,

$$
\alpha^{m}(g)=\alpha^{m-(k+1)}\left[g \prod_{r=1}^{k+1} b_{n-r}^{C(k+1, r)}\right]
$$

and the induction is complete. Now take $k=m=2^{n-1}$ and note that $\alpha^{0}=\iota$. Since $b$ 's with nonpositive subscript are $e$, we can write $\alpha^{m}(g)=g \prod_{r=1}^{m} b_{n-1}^{C(m, r)}$. By Lemma 15, $b_{n-r} \in \gamma_{\alpha}\left(B_{n-r+1}\right)$ implies that $b_{n-r}^{2^{n-r}}=e$. By Lemma 17 , however, $2^{n-r} \mid C(m, r)$, so that $\alpha^{m}(g)=g$.

Corollary. If $g \in B_{n}, n \geqq 2$, if $\alpha \in T_{1}$, and if $m=2^{n-1}$, then $\alpha^{m / 2}(g)$ $\equiv g \bmod B_{1}$, and $\gamma_{\alpha^{m / 2}}\left(g^{2}\right)=e$ In particular, if $G=B_{n}$, then $\alpha^{m / 2} \in V_{1}$, and $T_{1} / V_{1}$ is u.t. with exponent dividing $2^{n-2}$.

Proof. By Lemma 12, $g B_{1} \in B_{n-1}\left(G / B_{1}\right)$. Let $\alpha$ induce $\alpha^{\prime}$ on $G / B_{1}$. By the theorem, $\alpha^{\prime m / 2}\left(g B_{1}\right)=g B_{1}$, and $\alpha^{m / 2}(g) \equiv g \bmod B_{1}$; that is, $\alpha^{m / 2}(g)=g b$ where $b=\gamma_{\alpha^{m / 2}}(g) \in B_{1}$. Also by the theorem, $\alpha^{m}(g)=g$. But $\alpha^{m}(g)=\alpha^{m / 2}\left(\alpha^{m / 2}(g)\right)$ $=\alpha^{m / 2}(g b)=g b^{2}$, and $b^{2}=e$.

LEMMA 18. Let $n$ be an integer $\geqq 1$, and let $G$ be a group for which each automorphism of $G / B_{n}$ can be extended to a normal automorphism of $G$. Then if $\gamma_{\alpha}(g) \in B_{n}$ for every $\alpha \in T_{1}, g \in B_{n+1}$.

Proof. By hypothesis, $\alpha(g) \equiv g \bmod B_{n}$ for every $\alpha \in T_{1}$. Let $\alpha$ induce $\alpha^{\prime}$ on $G / B_{n} . \alpha^{\prime}\left(g B_{n}\right)=\alpha(g) B_{n}=g B_{n}$. Since the set of induced $\alpha^{\prime}$ coincides with $A\left(G / B_{n}\right)=T_{1}\left(G / B_{n}\right), g B_{n} \in B_{1}\left(G / B_{n}\right)=B_{n+1} / B_{n}$, and $g \in B_{n+1}$. 
9. The centralizer of $T_{1}$. Since $T_{1}$ is the centralizer of $J$ in $A$, $U_{1}=C\left(T_{1} ; A\right) \supset J$, where $U_{1}$ is a normal subgroup of $A$.

Lemma 19. (a) $B_{1}(G)$ is $U_{1}$-admissible, and if each automorphism of each $G / B_{i}(i=1,2,3, \cdots)$ can be extended to a normal automorphism of $G$, then each $B_{n}, n \geqq 2$, is likewise $U_{1}$-admissible. (b) $\gamma_{\alpha}\left(F\left(U_{1}\right)\right) \subset F\left(U_{1}\right) \subset Z_{1}$ for every $\alpha \in T_{1}$. (c) Each $\theta \in U_{1}$ induces an automorphism on each $F\left(\gamma_{\alpha}\right), \alpha \in T_{1}$.

Proof. (a) For $\theta \in U_{1}$ and $\alpha \in T_{1}, \theta \alpha(x)=\theta(x) \theta \gamma_{\alpha}(x)=\alpha \theta(x)=\theta(x) \gamma_{\alpha} \theta(x)$, so that $\theta \gamma_{\alpha}=\gamma_{\alpha} \theta$ for every $\alpha \in T_{1}$. If $g \in B_{1}$, then $\gamma_{\alpha} \theta(g)=\theta \gamma_{\alpha}(g)=\theta(e)=e$ for $\alpha \in T_{1}$, and $\theta(g) \in B_{1}$. Now suppose that $B_{n}$ is $U_{1}$-admissible. For $g \in B_{n+1}$, $\gamma_{\alpha} \theta(g)=\theta \gamma_{\alpha}(g) . \gamma_{\alpha}(g) \in B_{n}$, by Lemma 16. By the induction assumption, $\theta \gamma_{\alpha}(g) \in B_{n}$. Applying Lemma 18, $\theta(g) \in B_{n+1}$. (b) If $\theta(g)=g$ for every $\theta \in U_{1}$, then $\gamma_{\alpha} \theta(g)=\gamma_{\alpha}(g)=\theta \gamma_{\alpha}(g)$. Hence $\gamma_{\alpha}\left(F\left(U_{1}\right)\right) \subset F\left(U_{1}\right)$ for every $\alpha \in T_{1}$. If $\theta(g)=g$ for every $\theta \in U_{1}$, then $\tau_{x}(g)=g$ for every $x \in G$, since $J \subset U_{1}$. But $\tau_{x}(g)=g$ for every $x \in G$ implies that $g \in Z_{1}$. (c) If $g \in F\left(\gamma_{\alpha}\right), \gamma_{\alpha}(g)=g$, and $\theta \gamma_{\alpha}(g)=\gamma_{\alpha} \theta(g)=\theta(g)$, so that $\theta(g) \in F\left(\gamma_{\alpha}\right)$. Conversely, if $\theta(g) \in F\left(\gamma_{\alpha}\right)$, then $\theta \gamma_{\alpha}(g)=\gamma_{\alpha} \theta(g)=\theta(g)$. Since $\theta$ is an automorphism, $\gamma_{\alpha}(g)=g$, and $g \in F\left(\gamma_{\alpha}\right)$.

Theorem 5. Each element of $C\left(T_{1} ; A\right)$ induces a normal automorphism on $Z_{2}$, and there exists a homomorphism on $C\left(T_{1} ; A\right)$ into $T_{1}\left(Z_{2}\right)$ with kernel consisting of all those mappings in $C\left(T_{1} ; A\right)$ which reduce to the identity on $Z_{2}$.

Proof. $\theta \in U_{1}$ implies that $\theta$ commutes with every Grün automorphism of $G$. If, therefore, $u \in Z_{2}$, then $\theta\left(x^{-1} u^{-1} x u\right)=\theta\left(x^{-1}\right) \theta\left(u^{-1}\right) \theta(x) \theta(u)=\theta\left(x^{-1}\right) u^{-1} \theta(x) u$ for every $x \in G$. $u \theta\left(u^{-1}\right)$ is, consequently, in the centralizer of every $\theta(x)$, $x \in G$. Since $\theta$ is an automorphism, $u \theta\left(u^{-1}\right) \in Z_{1}(G) \subset Z_{1}\left(Z_{2}(G)\right)$, and $\theta(u)$ $\equiv u \bmod Z_{1}\left(Z_{2}(G)\right)$, so that $\theta$ restricted to $Z_{2}$ is normal thereon.

Corollary. If $G$ is of class 2 , then $J \subset C\left(T_{1} ; A\right) \subset T_{1}$, and $C\left(T_{1} ; A\right)$ $=Z_{1}\left(T_{1}\right)$.

10. The higher normal autmorphisms. If $\alpha \in A$ has the property $\alpha(x)$ $\equiv x \bmod Z_{n}$ for every $x \in G$, we say that $\alpha$ is an $n$-normal automorphism, and we have described the higher normal automorphisms of $G$. Let $T_{n}$ be the set of $n$-normal automorphisms of $G$. Under automorphism composition, $T_{n}$ is a normal subgroup of $G$, and $m \leqq n$ implies that $T_{m} \subset T_{n}$.

TheOREM 6. (a) $T_{n} / T_{n-1}$ is isomorphic to a subgroup of $T_{1}\left(G / Z_{n-1}\right)$. (b) $T_{n} / T_{1}$ is isomorphic to a subgroup of $T_{n}(J)$.

Proof. (a) $\alpha \in T_{n}$ induces an automorphism $\alpha^{\prime}$ on $G / Z_{n-1}$. For every $x \in G, x Z_{n-1} \in G / Z_{n-1}$, and $\alpha^{\prime}\left(x Z_{n-1}\right)=\alpha(x) Z_{n-1}=x z Z_{n-1}$ where $z \in Z_{n}$. Then $z Z_{n-1} \in Z_{1}\left(G / Z_{n-1}\right)$, so that $\alpha^{\prime}$ is normal on $G / Z_{n-1}$. It is not difficult to see that if $\alpha, \beta \in T_{n}$, then $(\alpha \beta)^{\prime}=\alpha^{\prime} \beta^{\prime}$, so that (') is a homomorphism on $T_{n}$ into $T_{1}\left(G / Z_{n-1}\right)$. Suppose that $\alpha^{\prime}=\imath$. Then $\alpha^{\prime}\left(x Z_{n-1}\right)=x Z_{n-1}$ for every $x \in G$, and 
$\alpha(x) \equiv x \bmod Z_{n-1}$. Hence $\alpha$ induces the identity on $G / Z_{n-1}$, and $\alpha \in T_{n-1}$. Conversely, if $\alpha \in T_{n-1}, \alpha^{\prime}\left(x Z_{n-1}\right)=\alpha(x) Z_{n-1}=x Z_{n-1}$, and then $\alpha^{\prime}=\iota$ on $G / Z_{n-1}$. Therefore, kern $\left(^{\prime}\right)=T_{n-1}$. (b) $\alpha \in T_{n+1}$ induces an automorphism $\alpha^{\prime \prime}$ on $G / Z_{1} \cong J$, given by $\alpha^{\prime \prime}\left(x Z_{1}\right)=\alpha(x) Z_{1}=x \gamma(x) Z_{1}$, where $\gamma(x) \in Z_{n+1}$. Hence $\alpha^{\prime \prime}\left(x Z_{1}\right)=x Z_{1} \bmod \left(Z_{n+1} / Z_{1}\right)$. Now $Z_{n}(J) \cong Z_{n}\left(G / Z_{1}\right) \cong Z_{n+1} / Z_{1}$, as an induction will show. Hence $\alpha^{\prime \prime}$ is, effectively, in $T_{n}(J) . \alpha$ induces $\iota$ if, and only if, $\alpha(x) \equiv x \bmod Z_{1}$ for every $x \in G$, and kern $\left({ }^{\prime \prime}\right)=T_{1}$.

CoRollary 1. Let $\alpha^{\prime}$ be a nontrivial normal automorphism of $G / Z_{n}$ which can be extended to a higher normal automorphism $\alpha$ of $G$. Then $\alpha \notin T_{n}$.

Corollary 2. Each $\alpha \in T_{n}$ induces a homomorphism of $G$ and an endomorphism of $G / Z_{n-1}$ into $Z_{n} / Z_{n-1}=Z_{1}\left(G / Z_{n-1}\right)$.

Proof. The endomorphism is $\gamma_{\alpha^{\prime}}$, and the homomorphism is obtained by following the natural mapping $\phi_{n-1}$ of $G$ onto $G / Z_{n-1}$ by $\gamma_{\alpha^{\prime}}$. Moreover, $\gamma_{\alpha^{\prime}} \phi_{n-1}(x)=x^{-1} \alpha(x) Z_{n-1}$ for every $x \in G$.

Let $S$ be a set of automorphisms of $G$ and let $N(S)$ be the set of all $g \in G$ such that $\alpha(g) \equiv g \bmod Z_{1}$ for every $\alpha \in S$.

Lemma 20. If $K$ is a subgroup of $A$, then $C(K ; A) \cap J=J(N(K) ; G)$. In particular, $Z_{1}(A) \cap J=J(N(A) ; G)$.

Proof. If $\tau_{o} \alpha=\alpha \tau_{g}$ for every $\alpha \in K$, then $g^{-1} \alpha(x) g=\alpha\left(g^{-1}\right) \alpha(x) \alpha(g)$ for every $x \in G$, so that $g \alpha\left(g^{-1}\right)$ is in the centralizer of every $\alpha(x)$. Since $\alpha$ is an automorphism, $g \alpha\left(g^{-1}\right) \in Z_{1}$, and $\alpha(g) \equiv g \bmod Z_{1}$, so that $g \in N(K)$ and $\tau_{g}$ $\in J(N(K) ; G)$. The proof can be read in reverse to obtain the converse.

Lemma 21. The following are equivalent: (a) $J \subset Z_{1}(A)$. (b) $A=T_{1}$. Either of these conditions implies that $G$ is of class 2.

Proof. $A=T_{1}$ if, and only if, $G=N(A)$. But if the latter holds, $J(N(A) ; G)$ $=J$; and conversely, if $J(N(A) ; G)=J, x \in G$ implies the existence of $y \in N(A)$ with $\tau_{x}=\tau_{y}$. Then $x \equiv y \bmod Z_{1}$, so that, if $\alpha \in A, \alpha(x) \equiv \alpha(y) \equiv y \equiv x \bmod Z_{1}$. This shows that $x \in N(A)$ and that $N(A)=G$. By Lemma 20, $J \cap Z_{1}(A)$ $=J(N(A) ; G)=J$, so that $J \subset Z_{1}(A)$, and (a) implies (b). A slight rearrangement of the above argument shows that (b) implies (a). Now if every automorphism of $G$ is a normal automorphism, $x^{-1} y x \equiv y \bmod Z_{1}$ for every $x, y \in G$. This implies that $G^{\prime} \subset Z_{1}$, and $G$ is of class 2 .

A similar result is contained in

Theorem 7. Let $G$ be a group with the properties (1) $J \subset T_{n}$ and (2) each $\alpha \in A$ induces $\iota$ on each $Z_{j+1} / Z_{j}(j=1,2, \cdots, n)$. Then $J \subset Z_{n}(A)$.

Proof. First, we establish three lemmas:

(R) For a group $G, J \subset T_{n}$ if, and only if, $G$ is of class $n+1$.

(S) For a group $G, J \cap Z_{n}(A) \subset J\left(Z_{n+1} ; G\right)$. 
(T) A group $G$ with property (2) has the further property that $J \cap Z_{n}(A)$ $=J\left(Z_{n+1} ; G\right)$ (for the $n$ of property (2)).

To prove (R), use the proof of the last statement of Lemma 21 as a model. As for (S), take $n=0$. Then $J \cap Z_{n}(A)$ consists of $\iota$ alone, and the inclusion is trivially valid. Suppose that it holds for $n=k$. $\tau_{\theta} \in J \cap Z_{k+1}(A)$ implies that $\left(\alpha, \tau_{g}\right) \in Z_{k}(A)$ for every $\alpha \in A$. A brief computation shows that $\left(\alpha, \tau_{g}\right)=\tau_{h}$, where $h=g \alpha^{-1}\left(g^{-1}\right)$. By the induction assumption, $g \alpha^{-1}\left(g^{-1}\right) \in Z_{k+1}$, and this is to be valid for every $\alpha \in A$. If we take $\alpha=\tau_{x}, x \in G$, then $\operatorname{gxg}^{-1} x^{-1} \in Z_{k+1}$ for every $x \in G$, and $g \in Z_{k+2}$. But this means that $J \cap Z_{k+1}(A) \subset J\left(Z_{k+2} ; G\right)$.

To prove $(\mathrm{T})$, let $\left\{\alpha_{i}\right\}(i=1,2, \cdots, n)$ be any finite set of elements of $A$. For a fixed $g \in G$, define $g_{1}=g \alpha_{1}^{-1}\left(g^{-1}\right)$. If $g_{k}$ is defined, let $g_{k+1}=g_{k} \alpha_{k+1}^{-1}\left(g_{k}^{-1}\right)$. A different finite set of elements of $A$, or even the same set in a different order, may very well lead to a different finite sequence $\left\{g_{i}\right\}$ on $g$. Let $G_{i}(g)$ $=G_{i}$ be the set of all $g_{i}$ obtained in this fashion for fixed $g$ and fixed positive integer $i$. By Lemma 20, $\tau_{\theta} \in Z_{1}(A)$ if, and only if, $g \in N(A)$. But $g \in N(A)$ if, and only if, $\alpha(g) \equiv g \bmod Z_{1}$ for every $\alpha \in A$. The latter rondition is equivalent to $G_{1} \subset Z_{1}$. Now suppose that $\tau_{g} \in Z_{k}(A)$ if, and only if, $G_{k} \subset Z_{1}$. $\tau_{g}$ $\in Z_{k+1}(A)$ if, and only if, $J\left(G_{1}(g) ; G\right) \subset Z_{k}(A)$. By the induction assumption, this is equivalent to $G_{k}(h) \subset Z_{1}$ for every $h \in G_{1}(g)$. Since $U G_{k}(h)=G_{k+1}(g)$, where the set union is taken over all $h \in G_{1}(g), \tau_{g} \in Z_{k+1}(A)$ if, and only if, $G_{k+1}(g) \subset Z_{1}$.

Now suppose that $\tau_{g} \in J\left(Z_{n+1} ; G\right)$. Then $g \in Z_{n+1}$ and $G_{1}(g) \subset Z_{n}$, since each $\alpha \in A$ induces the identity on $Z_{n+1} / Z_{n}$. Assume, inductively, that $G_{k}(g)$ $\subset Z_{n-k+1}$. Since each member of $A$ induces the identity on $Z_{n-k+1} / Z_{n-k}$, $G_{k+1}(g) \subset Z_{n-k}$. In particular, $G_{n}(g) \subset Z_{1}$. By the above, $J\left(Z_{n+1} ; G\right) \subset Z_{n}(A)$. Along with (S), this is enough to establish (T).

To prove the theorem, note that $J \subset T_{n}$ implies, by (R), that $G$ is of class $n+1$. Therefore, in (T), replace $Z_{n+1}$ by $G$. Since $J(G ; G)=J$, the theorem is proved.

For a subgroup $K$ of $A$, it is clear that $F(K) \subset N(K)$, that $Z_{1} \subset N(K)$, and that $N(K)$ is $K$-admissible. We prove a preliminary result on $Q(H ; G)$ for a characteristic subgroup $H$ of $G$.

Lemma 22. Let $H$ be a characteristic subgroup of $G$. (a) $Q(H ; G) \cap J$ $=J(H \div G ; G)$. (b) $\alpha$ and $\beta$ induce the same automorphism on $G / H$ if, and only if, $\alpha \equiv \beta \bmod Q(H ; G)$. In particular, $\tau_{x} \equiv \tau_{\nu}$ if, and only if, $x \equiv y \bmod H \div G$.

Proof. (a) $\tau_{g} \in Q(H ; G)$ if, and only if, $\tau_{g}(x) \equiv x \bmod H$ for every $\dot{x} \in G$. But this latter condition is equivalent to $\left(g, x^{-1}\right) \in H$ for every $x \in G$, and this is true if, and only if, $g \in H \div G$. (b) is obvious. $G)$.

Lemma 23. (a) $T_{n} \div J=T_{n+1}$. (b) $\left(T_{n}, J\right) \subset J\left(Z_{n} ; G\right)$. (c) $T_{n} \cap J=J\left(Z_{n+1}\right.$;

Proof. (a) and (b) can be established by routine arguments. To verify (c), 
replace $H$ by $Z_{n}$ in Lemma $22(\mathrm{a})$, and note that $Q\left(Z_{n} ; G\right)=T_{n}$ and that $Z_{n} \div G=Z_{n+1}$.

Corollary. (a) For a subgroup $K$ of $A$ and for a positive integer $n, N(K)$ $=Z_{n}$ if, and only if, $C(K ; A) \cap J=T_{n-1} \cap J$. (b) $N(K)=Z_{1}$ if, and only if, $C(K ; A) \cap J$ is trivial. (c) If $G$ is n-nilpotent, and if $K$ is a subgroup of $A$, then $K \subset T_{1}$ if, and only if, $C(K ; A) \cap J=T_{n-1} \cap J$.

Proof. (a) If $N(K)=Z_{n}(G)$, then $J\left(Z_{n} ; G\right)=J(N(K) ; G)=C(K ; A) \cap J$, by Lemma 20 . Since $J\left(Z_{n} ; G\right)=T_{n-1} \cap J$ (by (c) of the lemma), half the statement is established. Conversely, suppose that $C(K ; A) \cap J=T_{n-1} \cap J$ $=J\left(Z_{n} ; G\right)$. One can readily check the equivalence of the following statements: (1) $x \in Z_{n}$. (2) $\tau_{x} \alpha=\alpha \tau_{x}$ for every $\alpha \in K$. (3) $\tau_{x} \alpha \alpha^{-1}(y)=\alpha \tau_{x} \alpha^{-1}(y)$ for every $y \in G, \alpha \in K$. (4) $x^{-1} y x=\alpha\left(x^{-1}\right) y \alpha(x)$ for every $y \in G$ and every $\alpha \in K$. (5) $\alpha(x) x^{-1} \in Z_{1}$ for every $\alpha \in K$. (6) $x \in N(K)$. (b) follows from (a) by taking $n=1$. (c) $K \subset T_{1}$ if, and only if, $N(K)=G$. Since $G=Z_{n}$, (a) is applicable.

Theorem 8. $G^{(n)} \subset F\left(T_{n}\right) \subset C\left(Z_{n} ; G\right)$.

Proof. If $\alpha \in T_{n}$, then $\alpha\left(x^{-1} y^{-1} x y\right)=t^{-1} x^{-1} u^{-1} y^{-1} x t y u$ where $t, u \in Z_{n}$. Hence $\alpha\left(x^{-1} y^{-1} x y\right) \equiv x^{-1} y^{-1} x y \bmod Z_{n-1}$, and $\alpha$ induces the identity on $G^{\prime} /\left(Z_{n-1} \cap G^{\prime}\right)$. Suppose, inductively, that $\alpha \in T_{n}$ induces the identity on $G^{(k)} /\left(Z_{n-k} \cap G^{(k)}\right)$. A set of generators of $G^{(k+1)}$ is all $(x, y)$ where $x, y \in G^{(k)}$. $\alpha\left(x^{-1} y^{-1} x y\right)=t^{-1} x^{-1} u^{-1} y^{-1} x t y u$, where $t, u \in Z_{n-k}$. Hence $\alpha\left(x^{-1} y^{-1} x y\right) \equiv x^{-1} y^{-1} x y$ $\bmod Z_{n-k-1}$; and our induction shows that $\alpha \in T_{n}$ induces the identity on each $G^{(k)} /\left(Z_{n-k} \cap G^{(k)}\right)$. Now take $k=n$ so that $Z_{n-k}=(e)$. That is, each $\alpha \in T_{n}$ induces the identity on $G^{(n)}$, whence $G^{(n)} \subset F\left(T_{n}\right)$. By the discussion after Lemma $3, F\left(T_{n}\right) \subset C\left(Z_{n} ; G\right)$.

Corollary 1. $G^{(n)} \subset F\left(J\left(Z_{n+1} ; G\right)\right)$.

Proof. $T_{n} \supset J\left(Z_{n+1} ; G\right)$, by Lemma 23 (c).

CoRollaRy 2. If $F\left(T_{n}\right)=(e)$ or if $F\left(J\left(Z_{n+1} ; G\right)\right)=(e)$ for some positive integer $n$, then $G$ is solvable [3].

Corollary 3. $J\left(G^{(n)} ; G\right) \subset C\left(T_{n+1} ; A\right)$.

Proof. $\alpha \in T_{n+1}$, and $g \in G^{(n)}$ imply that $\alpha(g) \equiv g \bmod Z_{1}$, by the proof of the theorem. Hence $J\left(G^{(n)} ; G\right) \subset J\left(N\left(T_{n+1}\right) ; G\right)=C\left(T_{n+1} ; A\right) \cap J$, by Lemma 20.

If we let $U_{n}=C\left(T_{n} ; A\right)$, then, by Lemma $20, J\left(N\left(T_{n}\right) ; G\right) \subset U_{n}$. As in Lemma 19(a), $F\left(T_{n}\right)$ is $U_{n}$-admissible.

11. Examples. (A) For positive integers $n>2$, let $D_{n}$ denote the $n$th dihedral group, the group of isometries of a regular $n$-gon. $D_{n}$ is the semidirect product of $I_{n}$ and of $I_{2}$ with the multiplication rules $\left(x_{n}, 0_{2}\right)\left(y_{n}, z_{2}\right)$ $=\left(x_{n}+y_{n}, z_{2}\right)$ and $\left(x_{n}, 1_{2}\right)\left(y_{n}, z_{2}\right)=\left(x_{n}-y_{n}, 1_{2}+z_{2}\right)$. For $n>2$, there is a nontrivial element in the center if, and only if, $n$ is even; and in this case, the 
center consists of two elements, $\left(0_{n}, 0_{2}\right)$ and $\left(h_{n}, 0_{2}\right)$, where $h$ is an integer such that $2 h=n$. Since $D_{n}$ is a group with two generators, there are three nontrivial possibilities for central endomorphisms. The verification of the following results is easy: $T_{1}\left(D_{4 k}\right)$ is isomorphic to the Klein four group, $I_{2} \oplus I_{2}$. Let us denote the four group by $\mathfrak{B} . B_{1}\left(D_{4 k}\right)$ consists of all $\left(x_{4 k}, 0_{2}\right)$ where $x$ is even, so that $B_{1}\left(D_{4 k}\right) \cong I_{2 k}$. Likewise, $B_{1}\left(D_{4 k}\right)=D_{4 k}^{\prime}$, and, in fact, $D_{4 k} / B_{1}\left(D_{4 k}\right)$ $\cong \mathfrak{B}$. It follows that the $B$-series breaks off at $B_{1}\left(D_{4 k}\right) . T_{1}\left(D_{4 k+2}\right) \cong I_{2} . B_{1}\left(D_{4 k+2}\right)$ consists of all $\left(x_{4 k+2}, 0_{2}\right)$, so that $B_{1}\left(D_{4 k+2}\right) \cong I_{n}$. $D_{4 k+2} / B_{1}\left(D_{4 k+2}\right) \cong I_{2}$ so that, by Lemma $11, D_{4 k+2}$ is $2-B$-nilpotent. If $n=4$, then $D_{4} / Z_{1}\left(D_{4}\right)$ is isomorphic to $\mathfrak{B}$ whence $D_{4}$ is of class 2 . Then $T_{2}\left(D_{4}\right)=A\left(D_{4}\right)$, and it can be readily verified that $A\left(D_{4}\right) \cong D_{4}$ and that $F\left(T_{2}\left(D_{4}\right)\right)=B_{1}\left(D_{4}\right)=Z_{1}\left(D_{4}\right)$.

(B) Let $G$ be a group of type $\left(2^{\infty}\right) . G$ is isomorphic to the additive group, modulo 1 , of the rationals $k / 2^{n}$, where $k$ is an odd integer or 0 . Since $G$ is abelian, $T_{1}(G)=A(G)$. $G$ has a nontrivial automorphism $\alpha\left(k / 2^{n}\right)=1-\left(k / 2^{n}\right)$ corresponding to the conjugation automorphism on the representation of $G$ on the unit circle. The only fixed points are $1=0$ and $1 / 2$. Conversely, if $\beta$ is any automorphism of $G, 2 \beta(1 / 2)=\beta(1)=1=0$, so that $\beta(1 / 2)=1 / 2$ or 0 . Thus $B_{1} \cong I_{2}$. Since $G / B_{1} \cong G, B_{2}$ consists of $0,1 / 4,1 / 2$, and $3 / 4$, and $B_{2} \cong I_{4}$. In general, $B_{n} \cong I_{2^{n}}$. $G=\bigcup B_{n}$ where the union is taken over all positive integral values of $n$.

(C) Let $G$ be the multiplicative group of all nonsingular 2 by 2 matrices over the field of rationals, $R$. It is well known that $Z_{1}$ consists of all

$$
\left(\begin{array}{ll}
u & 0 \\
0 & u
\end{array}\right), \quad u \neq 0,
$$

and that $Z_{2}=Z_{1}$. By Lemma 1 , we have an example of a group for which $T_{1} \cap J$ is trivial. Let $\mu$ be an endomorphism of the multiplicative group of nonzero rationals $R^{*}$ where $x \mu\left(x^{2} a\right)=1$ has a unique solution $x=x(a ; \mu)$ for every $a \in R^{*}$. Let $d_{1}$ and $d_{2}$ be integers with the restriction $\left|d_{i}\right|=1$. Define a mapping $\alpha=\alpha\left(\mu ; d_{1}, d_{2}\right)$ on $G$ by

$$
\begin{gathered}
\alpha\left(\begin{array}{ll}
r & 0 \\
0 & 1
\end{array}\right)=\left(\begin{array}{cc}
r \mu(r) & 0 \\
0 & \mu(r)
\end{array}\right) \quad \text { for every } r \in R^{*}, \\
\alpha\left(\begin{array}{ll}
0 & 1 \\
1 & 0
\end{array}\right)=\left(\begin{array}{ll}
0 & d_{1} \\
d_{1} & 0
\end{array}\right) \quad \text { and } \alpha\left(\begin{array}{ll}
1 & 1 \\
0 & 1
\end{array}\right)=\left(\begin{array}{ll}
d_{2} & d_{2} \\
0 & d_{2}
\end{array}\right) .
\end{gathered}
$$

Then it is possible to prove that $\alpha$ is a normal automorphism of $G$, and each normal automorphism of $G$ is such an $\alpha\left(\mu ; d_{1}, d_{2}\right)$. A matrix $M \in G$ is in $B_{1}$ if, and only if, it can be factored (without regard to the order of the factors) into a product of an even number of factors

$$
\left(\begin{array}{ll}
1 & 1 \\
0 & 1
\end{array}\right),
$$


an even number of factors

$$
\left(\begin{array}{ll}
0 & 1 \\
1 & 0
\end{array}\right)
$$

and a set of factors

$$
\left(\begin{array}{ll}
a_{i} & 0 \\
0 & 1
\end{array}\right) \quad(i=1,2, \cdots, n)
$$

where $a_{1} a_{2} \cdots a_{n}=1 . V_{1}$ turns out to be the set of all $\alpha\left(\mu ; d_{1}, d_{2}\right)$ with $\mu(x)= \pm 1$ for every $x \in R^{*}$. $W_{1}^{*}$ consists of all $\alpha$ with $\mu(x)= \pm(1 / x)$, and $\left[T_{1}: V_{1}\right]$ is equal to the number of normal subgroups of index 2 in $G$ which contain

$$
\left(\begin{array}{rr}
-1 & 0 \\
0 & -1
\end{array}\right)
$$

$W_{1}$ for this group is abelian. Now $W_{1}^{*}$ is nonvoid, $V_{1}$ is nontrivial and $Z_{1}$ has the periodic element

$$
\left(\begin{array}{rr}
-1 & 0 \\
0 & -1
\end{array}\right)
$$

Therefore, this example shows that we cannot drop the hypothesis of aperiodicity for $Z_{1}$ in Theorem 1 , Corollary $1(\mathrm{~b}) . G / B_{1} \cong \mathfrak{B} \oplus R^{*}$ whence $B_{2}=B_{1}$. Let $\mu$ be an endomorphism of $R^{*}$ such that, to each positive prime $p$, there exists a positive prime $q$ with $q|\mu(p)|=1=q|\mu(q)|$. Then $\alpha\left(\mu ; d_{1}, d_{2}\right) \in T_{1}$, so that, for this group, $T_{1}$ is far from trivial and $T_{1} \neq W_{1}$. Negatively, one can show, for instance, that if $\mu$ is an endomorphism of $R^{*}$ for which $|\mu(p)|$ is always a product of $k$ positive primes (or a product of the reciprocals of $k+1$ positive primes) for every positive prime $p$, then the corresponding $\alpha$ is not an automorphism.

(D) Let $G$ be a group with generators $a, b$, and $c$, where $a^{2}=e, a b=b a$, $a c=c a$, and $b c=c b a$. Then every element of $G$ can be written uniquely as a product $a^{i} b^{j} c^{k}$ where $i$ is 0 or 1 , and $j$ and $k$ range over the integers. $Z_{1} \cong I_{2}$ and $G / Z_{1} \cong R \oplus R$ so that $G$ is nilpotent of class 2 . One can verify that $T_{1} \cong \mathfrak{B}$. An element is in $B_{1}$ if, and only if, $j$ and $k$ are both even. Under any automorphism $\alpha$ each center element, $a^{i}$, is fixed. There is an automorphism $\beta$ which changes the sign of $j$ in each term. Its set of fixed points is precisely all elements with $j=0$. There is an automorphism $\delta$ which changes the sign of $k$ in each term, and the corresponding fixed points are all elements with $k=0$. The cross-cut of these two sets of fixed points is $Z_{1}$, so that $Z_{1}=F(A)$ $=F\left(T_{2}\right)$, and this latter set is included in $F\left(T_{1}\right)=B_{1}$ properly. (In the example of $D_{4}$ above, $F\left(T_{1}\right)=F\left(T_{2}\right)$ for the class 2 group $D_{4}$.) 
Presumably, by extending the grot $\mathrm{p}$ of this example or by considering $n$ by $n$ triangular matrices with a diagonal of unities, one could exhibit groups with significant $T_{n}$, for $n>2$.

\section{BIBLIOGRAPHY}

1. N. J. S. Hughes, The structure and order of the group of central automorphisms of a finite group, Proc. London Math. Soc. (2) vol. 52 (1951) pp. 377-385.

2. A. Kuros, Teoriia Grupp, Moscow-Leningrad, 1944.

3. H. Zassenhaus, Gruppentheorie, Leipzig-Berlin, 1937. (References are to the 1949 English translation.)

WASHINGTON UNIVERSITY,

SaINT Louis, Mo. 